\title{
Choice of Basis Functions for Accurate Characterization of Infinite Array of Microstrip Reflectarray Elements
}

\author{
Sembiam R. Rengarajan, Fellow, IEEE
}

\begin{abstract}
The integral equation of an infinite array of microstrip elements in the form of patches or crossed dipoles excited by a uniform plane wave is solved by the method of moments. Efficient choices of entire domain basis functions that yield accurate results are investigated.
\end{abstract}

Index Terms-Basis functions, infinite arrays, method of moments, microstrip, reflectarrays.

\section{INTRODUCTION}

$\mathbf{M}$ ICROSTRIP reflectarrays are used in many spacecraft communication and radar systems because of their low profile and ease of deployment. In the analysis and design of reflectarrays one usually uses the reflection coefficient of the canonical geometry of an infinite array of identical periodic elements excited by a uniform plane wave [1]. This approximation is based on the fact that in reflectarrays the size and shape of the microstrip elements vary slowly as a function of distance. A local plane wave model for the feed illumination is a good assumption. The problem of an infinite array of microstrip elements is formulated in terms of an integral equation for the unknown currents in the microstrip element of a unit cell. The Green function in the integral equation consists of a double summation of Floquet modes. The integral equation is usually solved by the method of moments (MoM). Entire domain basis functions are preferred in the moment method for microstrip elements such as rectangular patches and dipoles [1], [2]. We investigated the effects of the number and type of basis functions of the moment method on the reflection phase of the microstrip reflectarrays. The objective of this work was to identify basis functions that provide efficient and accurate solutions for the induced patch currents and the reflection phase.

\section{METHOD}

The method of analysis and computation is essentially the same as the MoM technique discussed in [1], [2], the only difference being the choice of basis functions in this paper. Dielectric losses are computed by using complex values of permittivity for the substrate materials while conductor losses are de-

Manuscript received November 10, 2004; revised December 16, 2004. The research described in this paper was conducted at the Jet Propulsion Laboratory, California Institute of Technology, Pasadena, CA, under a contract with the National Aeronautics and Space Administration.

The author is with the California State University, Northridge, CA 913308346 USA and also with the Jet Propulsion Laboratory, California Institute of Technology, Pasadena, CA 91109 USA.

Digital Object Identifier 10.1109/LAWP.2005.844127 termined by a perturbation method. All the basis functions used in this work and their Fourier transforms are listed in the Appendix. The Green function used in this work is the same as that given in equation (11) in [1]. In all the numerical computations to be discussed we typically used about 7200 Floquet modes. The trigonometric basis functions (1) through (8) in Appendix I have been widely used in the literature and they form a complete set of independent functions with the boundary conditions that the current perpendicular to an edge is zero at the edge and it is a positive or negative maximum at a parallel edge. These functions are slow to converge and we did not obtain adequate accuracy even with the use of many basis functions. The principal reason for the slow convergence of the trigonometric basis functions is that they do not enforce the correct edge conditions. Two entire domain basis functions exhibiting the appropriate edge conditions along and across the current direction, shown in (9) through (12) in Appendix I, are $\phi_{1}(x, a)$ and $\phi_{2}(y, b)$ respectively for $\mathrm{J}_{x}$. Trigonometric basis functions with multiplicative edge conditions discussed in (13) through (20) in Appendix I also form a complete set and they exhibit the correct edge conditions for thin patches [3]. In addition, their Fourier transforms are closed form expressions. These basis functions exhibit excellent convergence properties. We examined the convergence and accuracy of the solutions by using as many as 512 basis functions, i.e., eight terms in each series in (23) of Appendix II. Unless the substrate is extremely thin we found good convergence for 32 terms in (23). For crossed dipoles Pozar employs a crooked or bent mode of current given in (24) in Appendix II. Such a function was used in frequency selective surfaces employing the Jerusalem Cross shape [4]. Munk has observed that in the case of crossed dipoles excitation of such a mode of current is not significant. Numerical results evaluated for the reflection amplitude and phase in this work were compared to waveguide simulator experimental data and results computed from the commercial code HFSS from ANSOFT corporation. The waveguide simulator is similar to the one used by Pozar [5] with two patches along the broad dimension of the waveguide. In this investigation we used square patches and crossed dipoles for use in dual polarization and circular polarization applications. The results to be presented in this paper are also applicable to rectangular patches and dipole arrays for linear polarization.

\section{RESUlTS AND DISCUSSION}

Fig. 1 shows a comparison of measured and computed (labeled SR1 with loss in the figure) data for the reflection 


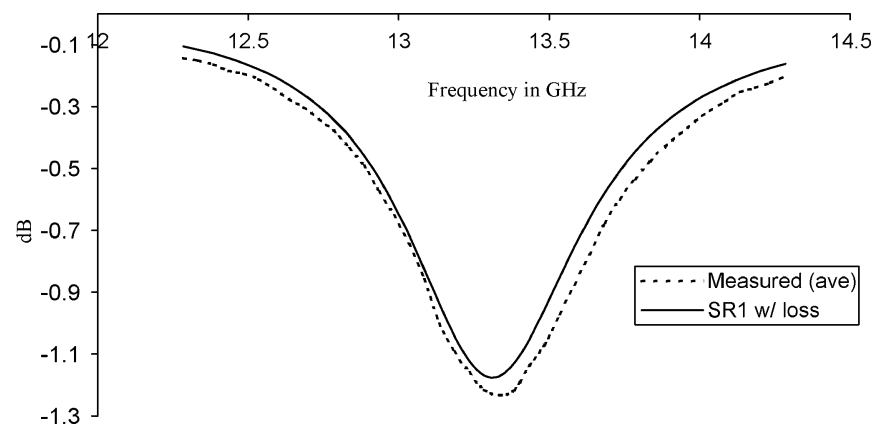

Fig. 1. Reflection coefficient magnitude in decibels versus frequency of measured data and computed using the basis functions (22) in Appendix II.

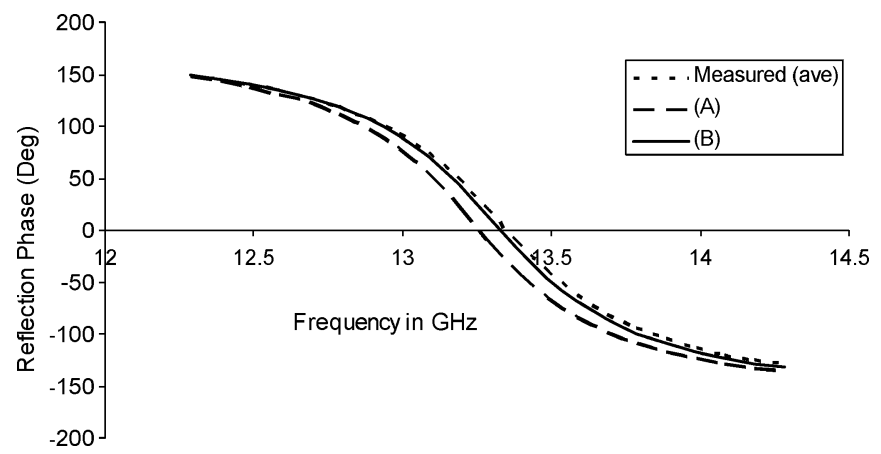

Fig. 2. Reflection coefficient phase versus frequency for measured and computed data. (A) Basis functions (21). (B) Basis functions (22).

coefficient magnitude in decibels versus frequency for a reflectarray of square patches. The unit cell is a square of side equal to a half free-space wavelength at $13.285 \mathrm{GHz}$. The patch size is $5.414 \mathrm{~mm}$. The substrate dielectric constant is 3.58 and the loss tangent is 0.0029 . The dielectric thickness is $0.081 \mathrm{~cm}$. The two sets of data agree within $0.1 \mathrm{~dB}$. Figs. 1 and 2 correspond to a $30^{\circ}$ scan in the H-plane. The reflection phase data are shown in Fig. 2. The choice of basis functions in (21) in Appendix II yields reasonably good agreement with measurements whereas the choice (22) provides excellent agreement. The use of multiplicative edge conditions employed in (22) is a more accurate representation of the current distribution than the additive edge condition employed in (21). The measurement data are averaged over a few coupons of nominal dimensions stated above. The use of edge conditions in the transverse direction, not shown here, provided poor results. The reason for this is clear from an examination of Fig. 3 that illustrates the transverse distribution of patch current computed by the HFSS. Because of symmetry only half of the plot is shown. The size of the patch is different in this case but it is at resonance. The distribution is uniform in the transverse direction except very close to the edges. When the edge condition is employed in the transverse direction with only a few basis functions a rather large error in much of the transverse aperture is introduced.

For reduced values of the substrate thickness, moment method computations with a few basis functions do not provide good accuracy. Thinner substrates result in higher $\mathrm{Q}$ patches, for which the reflection phase at resonance exhibits greater sensitivity to the choice of basis functions. Such patches require many more basis functions such as the series (23). Fig. 4 illustrates the reflection phase values for an array similar to
Normalized current in transverse direction at resonance from HFSS

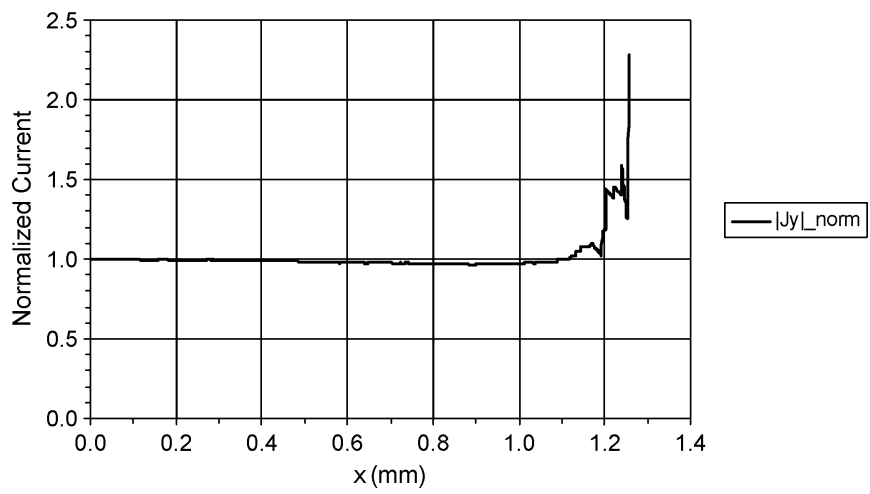

Fig. 3. Normalized current distribution across the current flow direction (computed by HFSS).

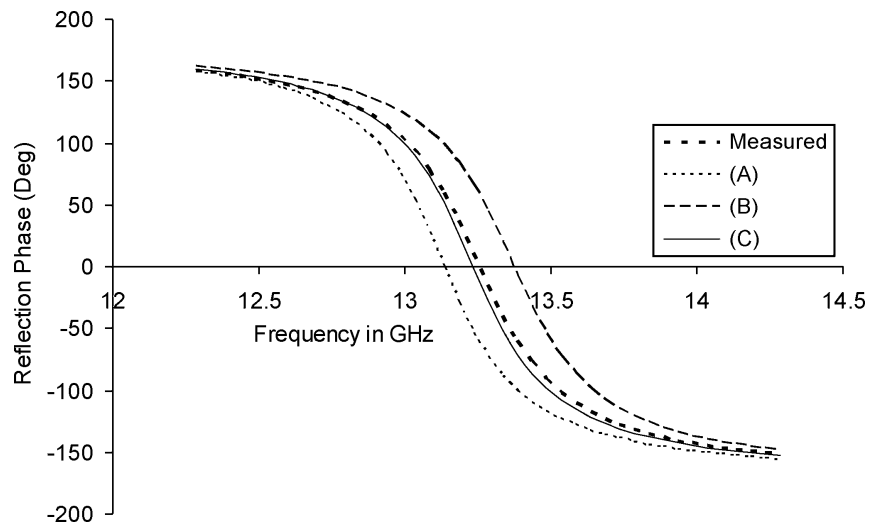

Fig. 4. Reflection phase versus frequency for a reflectarray of square patches on a thin substrate. (A) Basis functions (21). (B) Basis functions (22). (C) Basis functions (23).

that in Figs. 1 and 2 but for a patch size of $5.668 \mathrm{~mm}$ and a dielectric thickness of $0.0508 \mathrm{~cm}$. Once again an H-plane scan of $30^{\circ}$ is considered. In this case the moment method results for basis functions (21) and (22) did not yield good accuracy whereas the choice of 32 basis functions (23) yielded excellent agreement with measurements. The use of MoM code with one basis function for each current such as (22) or (25) is extremely fast and useful in the analysis and design of large reflectarrays. Even a MoM code with 32 basis functions such as (23) is much faster than HFSS. General purpose codes such as HFSS are slow and, hence, are impractical to use in direct analysis and design applications.

In Fig. 5 we compare the reflection phase data computed by HFSS and moment method with choice of basis functions (24) and (25) for an array of crossed dipoles for a normally incident plane wave at $7.115 \mathrm{GHz}$. Our investigations showed that the amplitude of excitation of crooked or bent mode current in (24) is not significant. In the case of dipoles the edge condition transverse to the dipole axis is very significant whereas the edge condition along the dipole is unnecessary. The dipole is resonant at $7.115 \mathrm{GHz}$ for a length of $18.66 \mathrm{~mm}$. The width of the dipole is $1.5 \mathrm{~mm}$. The substrate consists of two layers with dielectric constant, loss tangent, and thickness of 1.093, 0.0027, $0.165 \mathrm{~cm}$, 


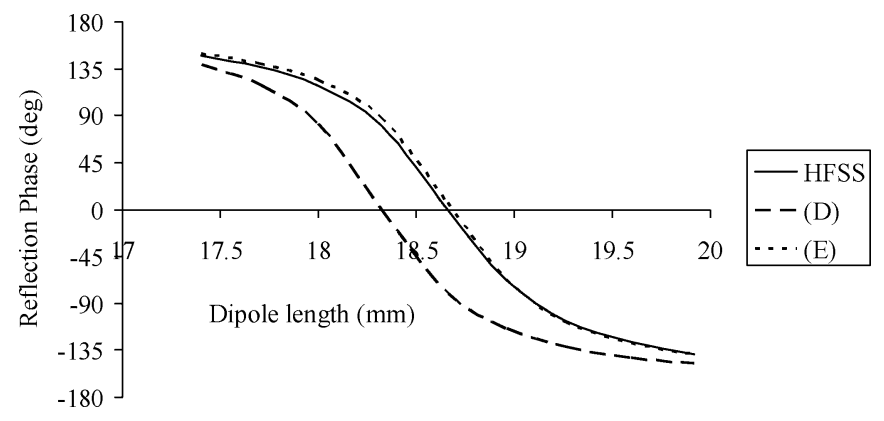

Fig. 5. Reflection phase versus dipole length for a reflectarray of crossed dipoles. (D) Basis functions (24). (E) Basis functions (25).

$3.0,0.0013$, and $0.0305 \mathrm{~cm}$ at the bottom and top layers, respectively. The unit cell square size is 0.552 wavelength in free space. Clearly the choice of basis functions (25) employing the edge conditions in the transverse direction yields very accurate results. The HFSS data were carefully computed with sufficient number of tetrahedra until good convergence was obtained. For small values of the substrate thickness, we needed many basis functions for good convergence, a result similar to that in Fig. 4 for patch arrays.

\section{CONCLUSION}

For a substrate thickness greater than 0.065 wavelength in the dielectric, basis functions (22) and (25) yield excellent accuracy for patches and dipoles, respectively. A choice of 32 trigonometric basis functions (23), all incorporating multiplicative edge conditions provide very good results for substrates of thickness about 0.04 wavelength in the dielectric. For extremely thin substrates in the order of 0.02 wavelength the resonant microstrip element exhibits high $\mathrm{Q}$ and, hence, the convergence of the solution becomes poor. The HFSS results are also poor to converge in terms of the required number of tetrahedra. Based on considerations of accurate computation of reflection phase of microstrip elements near resonance and design tolerances we recommend that the substrate thickness be chosen greater than 0.04 wavelength in the dielectric.

\section{APPENDIX I}

BASIS FUNCTIONS AND THEIR FOURIER TRANSFORMS

$$
\begin{aligned}
& \mathrm{f}_{1 \mathrm{e}}(x, a, m)= \cos \left(\frac{\mathrm{m} \pi \mathrm{x}}{\mathrm{a}}\right) \\
& \mathrm{m}=1,3,5 \ldots-\frac{\mathrm{a}}{2} \leq \mathrm{x} \leq \frac{\mathrm{a}}{2} \\
& \mathrm{~F}_{1 \mathrm{e}}\left(k_{x}, a, m\right)=(-1)^{\frac{(\mathrm{m}-1)}{2}} 2\left(\frac{\mathrm{m} \pi}{\mathrm{a}}\right) \cos \left(\frac{k_{x} \mathrm{a}}{2}\right) \\
& \times\left[\left(\frac{\mathrm{m} \pi}{\mathrm{a}}\right)^{2}-k_{x}^{2}\right]^{-1} . \\
& \mathrm{f}_{1 \mathrm{o}}(x, a, m+1)= \sin \left[\frac{(\mathrm{m}+1) \pi \mathrm{x}}{\mathrm{a}}\right] \\
& \mathrm{m}=1,3,5 \ldots-\frac{\mathrm{a}}{2} \leq \mathrm{x} \leq \frac{\mathrm{a}}{2}
\end{aligned}
$$

$$
\begin{aligned}
& \mathrm{F}_{1 \mathrm{o}}\left(k_{x}, a, m+1\right)=\mathrm{j}(-1)^{\frac{(\mathrm{m}+1)}{2}} 2(\mathrm{~m}+1)\left(\frac{\pi}{\mathrm{a}}\right) \sin \left(\frac{k_{x} \mathrm{a}}{2}\right) \\
& \times\left[\left\{\frac{(\mathrm{m}+1) \pi}{\mathrm{a}}\right\}^{2}-k_{x}^{2}\right]^{-1} . \\
& \mathrm{f}_{2 \mathrm{e}}(y, b, n)=\cos \left(\frac{\mathrm{n} \pi \mathrm{y}}{\mathrm{b}}\right) \\
& \mathrm{n}=0,2,4 \ldots \quad-\frac{\mathrm{b}}{2} \leq \mathrm{y} \leq \frac{\mathrm{b}}{2} \\
& \mathrm{~F}_{2 \mathrm{e}}\left(k_{y}, b, n\right)=-(-1)^{\frac{\mathrm{n}}{2}} 2 k_{y} \sin \left(\frac{k_{y} \mathrm{~b}}{2}\right) \\
& \times\left[\left(\frac{\mathrm{n} \pi}{\mathrm{b}}\right)^{2}-k_{y}^{2}\right]^{-1} .
\end{aligned}
$$

$$
\mathrm{f}_{2 \mathrm{o}}(y, b, n+1)=\sin \left[\frac{(\mathrm{n}+1) \pi \mathrm{y}}{\mathrm{b}}\right]
$$$$
\mathrm{n}=0,2,4 \ldots-\frac{\mathrm{b}}{2} \leq \mathrm{y} \leq \frac{\mathrm{b}}{2}
$$$$
\mathrm{F}_{2 \mathrm{o}}\left(k_{y}, b, n\right)=\mathrm{j}(-1)^{\frac{(\mathrm{n}+2)}{2}} 2 k_{y} \cos \left(\frac{k_{y} \mathrm{~b}}{2}\right)
$$$$
\times\left[\left\{\frac{(\mathrm{n}+1) \pi}{\mathrm{b}}\right\}^{2}-k_{y}^{2}\right]^{-1} .
$$

$$
\begin{aligned}
\Phi_{1}(x, a) & =\left[1-\left(\frac{2 \mathrm{x}}{\mathrm{a}}\right)^{2}\right]^{\frac{1}{2}}-\frac{\mathrm{a}}{2} \leq \mathrm{x} \leq \frac{\mathrm{a}}{2} \\
\tilde{\Phi}_{1}\left(k_{x}, a\right) & =\frac{\pi}{\mathrm{k}_{\mathrm{x}}} J_{1}\left(\frac{\mathrm{k}_{\mathrm{x}} a}{2}\right) .
\end{aligned}
$$$$
\Phi_{2}(y, b)=\left[1-\left(\frac{2 \mathrm{y}}{\mathrm{b}}\right)^{2}\right]^{-\frac{1}{2}} \quad-\frac{\mathrm{b}}{2} \leq \mathrm{y} \leq \frac{\mathrm{b}}{2}
$$$$
\tilde{\Phi}_{2}\left(k_{y}, b\right)=\frac{\pi b}{2} J_{0}\left(\frac{\left|\mathrm{k}_{\mathrm{y}}\right| \mathrm{b}}{2}\right) \text {. }
$$

$$
\begin{aligned}
\mathrm{g}_{1 \mathrm{e}}(x, a, m)= & \cos \left(\frac{\mathrm{m} \pi \mathrm{x}}{\mathrm{a}}\right)\left[1-\left(\frac{2 \mathrm{x}}{\mathrm{a}}\right)^{2}\right]^{-\frac{1}{2}} \\
\mathrm{~m}= & 1,3,5, \ldots-\frac{\mathrm{a}}{2} \leq \mathrm{x} \leq \frac{\mathrm{a}}{2} \\
\mathrm{G}_{1 \mathrm{e}}\left(k_{x}, a, m\right)=\frac{\pi \mathrm{a}}{4}[ & J_{0}\left(\frac{a}{2}\left|k_{x}+\left(\frac{m \pi}{a}\right)\right|\right) \\
& \left.+J_{0}\left(\frac{a}{2}\left|k_{x}-\left(\frac{m \pi}{a}\right)\right|\right)\right] .
\end{aligned}
$$

$$
\begin{aligned}
& \mathrm{g}_{1 \mathrm{o}}(x, a, m+1) \\
& =\sin \left[\frac{(\mathrm{m}+1) \pi \mathrm{x}}{\mathrm{a}}\right]\left[1-\left(\frac{2 \mathrm{x}}{\mathrm{a}}\right)^{2}\right]^{-\frac{1}{2}} \\
& \mathrm{~m}=1,3,5, \ldots \quad-\frac{\mathrm{a}}{2} \leq \mathrm{x} \leq \frac{\mathrm{a}}{2} \\
& \mathrm{G}_{1 \mathrm{o}}\left(k_{x}, a, m+1\right) \\
& =\frac{\pi \mathrm{a}}{\mathrm{j} 4}\left[J_{0}\left(\frac{a}{2}\left|k_{x}-\frac{(m+1) \pi}{a}\right|\right)\right. \\
& \left.\quad-J_{0}\left(\frac{a}{2}\left|k_{x}+\frac{(m+1) \pi}{a}\right|\right)\right] .
\end{aligned}
$$




$$
\begin{aligned}
\mathrm{g}_{2 \mathrm{e}}(y, b, n)=\cos \left(\frac{\mathrm{n} \pi \mathrm{y}}{\mathrm{b}}\right)\left[1-\left(\frac{2 \mathrm{y}}{\mathrm{b}}\right)^{2}\right]^{-\frac{1}{2}} \\
\mathrm{n}=0,2,4, \ldots \quad-\frac{\mathrm{b}}{2} \leq \mathrm{y} \leq \frac{\mathrm{b}}{2} \\
\mathrm{G}_{2 \mathrm{e}}\left(k_{y}, b, n\right)=\frac{\pi \mathrm{b}}{4}\left[J_{0}\left(\frac{b}{2}\left|k_{y}+\left(\frac{n \pi}{\mathrm{b}}\right)\right|\right)\right. \\
\left.+J_{0}\left(\frac{b}{2}\left|k_{y}-\left(\frac{n \pi}{b}\right)\right|\right)\right] . \quad \\
\mathrm{g}_{2 \mathrm{o}}(y, b, n+1)=\sin \left[\frac{(\mathrm{n}+1) \pi \mathrm{y}}{\mathrm{b}}\right]\left[1-\left(\frac{2 \mathrm{y}}{\mathrm{b}}\right)^{2}\right]^{-\frac{1}{2}} \\
\mathrm{n}=0,2,4, \ldots-\frac{\mathrm{b}}{2} \leq \mathrm{y} \leq \frac{\mathrm{b}}{2} \\
\mathrm{G}_{2 \mathrm{o}}\left(k_{y}, b, n+1\right)=\frac{\pi \mathrm{b}}{\mathrm{j} 4}\left[J_{0}\left(\frac{b}{2}\left|k_{y}-\frac{(n+1) \pi}{b}\right|\right)\right. \\
\left.-J_{0}\left(\frac{b}{2}\left|k_{y}+\frac{(n+1) \pi}{b}\right|\right)\right]
\end{aligned}
$$

where $J_{0}$ and $J_{1}$ are Bessel functions of the first kind and orders 0 and 1 , respectively.

\section{APPENDIX II}

The following basis functions of currents in patches and dipoles refer to Figs. 6 and 7 and various space domain functions and Fourier transforms given in (1) through (20).

1) Pozar's basis functions for the rectangular patch currents

$$
\begin{aligned}
& J_{x}(x, y)=\left[\mathrm{A}_{1} \mathrm{f}_{1 \mathrm{e}}(x, a, 1)+\mathrm{B}_{1} \Phi_{1}(x, a)\right] \mathrm{f}_{2 \mathrm{e}}(y, b, 0) \\
& J_{y}(x, y)=\left[\mathrm{C}_{1} \mathrm{f}_{1 \mathrm{e}}(y, b, 1)+\mathrm{D}_{1} \Phi_{1}(y, b)\right] \mathrm{f}_{2 \mathrm{e}}(x, a, 0) .
\end{aligned}
$$

2) Efficient basis functions for patch currents

$$
\begin{aligned}
& J_{x}(x, y)=\mathrm{A}_{1} \mathrm{~g}_{1 \mathrm{e}}(x, a, 1) \mathrm{f}_{2 \mathrm{e}}(y, b, 0) \\
& J_{y}(x, y)=\mathrm{B}_{1} \mathrm{~g}_{1 \mathrm{e}}(y, b, 1) \mathrm{f}_{2 \mathrm{e}}(x, a, 0) .
\end{aligned}
$$

3) Basis functions for accurate characterization of currents

$$
\begin{aligned}
J_{x}(x, y) & {\left[\sum_{\mathrm{m}=1,3} \mathrm{~A}_{\mathrm{m}} \mathrm{g}_{1 \mathrm{e}}(x, a, m)+\mathrm{B}_{\mathrm{m}} \mathrm{g}_{1 \mathrm{o}}(x, a, m+1)\right] } \\
& \times\left[\sum_{\mathrm{n}=0,2} \mathrm{C}_{\mathrm{n}} \mathrm{g}_{2 \mathrm{e}}(y, b, n)+\mathrm{D}_{\mathrm{n}} \mathrm{g}_{2 \mathrm{o}}(y, b, n+1)\right] \\
J_{y}(x, y) & {\left[\sum_{\mathrm{m}=1,3,} \mathrm{~A}_{\mathrm{m}}^{\prime} \mathrm{g}_{1 \mathrm{e}}(y, b, m)+\mathrm{B}_{\mathrm{m}}^{\prime} \mathrm{g}_{1 \mathrm{o}}(y, b, m+1)\right] } \\
& \times\left[\sum_{\mathrm{n}=0,2} \mathrm{C}_{\mathrm{n}}^{\prime} \mathrm{g}_{2 \mathrm{e}}(x, a, n)+\mathrm{D}_{\mathrm{n}}^{\prime} \mathrm{g}_{2 \mathrm{o}}(x, a, n+1)\right] .
\end{aligned}
$$

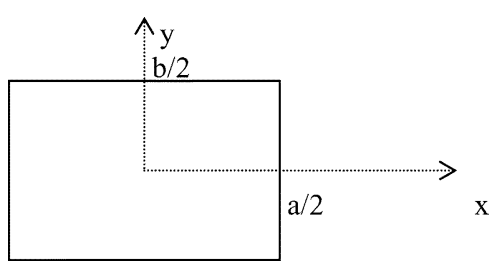

Fig. 6. Rectangular patch $(\mathrm{a} \times \mathrm{b})$.

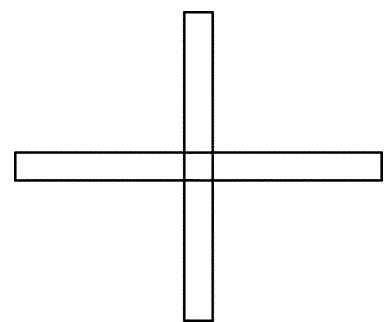

Fig. 7. Crossed dipoles (length $=\mathrm{a}$, width $=\mathrm{b})$.

4) Pozar's basis functions for dipoles

$$
\begin{aligned}
& J_{x}(x, y)=\left[\mathrm{A}_{1} \mathrm{f}_{1 \mathrm{e}}(x, a, 1)+\mathrm{A}_{2} \Phi_{1}(x, a)\right] \mathrm{f}_{2 \mathrm{e}}(y, b, 0) \\
& J_{y}(x, y)=\left[\mathrm{B}_{1} \mathrm{f}_{1 \mathrm{e}}(y, a, 1)+\mathrm{B}_{2} \Phi_{1}(y, a)\right] \mathrm{f}_{2 \mathrm{e}}(x, b, 0) \\
& \vec{J}_{c}(x, y)=\mathrm{C}_{1}\left[\hat{\mathrm{x}} \operatorname{sgn}(\mathrm{x}) \mathrm{f}_{1 \mathrm{e}}(x, a, 1) \mathrm{f}_{2 \mathrm{e}}(y, b, 0)\right. \\
&\left.-\hat{\mathrm{y}} \operatorname{sgn}(\mathrm{y}) \mathrm{f}_{1 \mathrm{e}}(y, a, 1) \mathrm{f}_{2 \mathrm{e}}(x, b, 0)\right] .
\end{aligned}
$$

5)

Efficient basis functions for dipole currents

$$
\begin{aligned}
& J_{x}(x, y)=\mathrm{A}_{1} \mathrm{f}_{1 \mathrm{e}}(x, a, 1) \mathrm{g}_{2 \mathrm{e}}(y, b, 0) \\
& J_{y}(x, y)=\mathrm{B}_{1} \mathrm{f}_{1 \mathrm{e}}(y, a, 1) \mathrm{g}_{2 \mathrm{e}}(x, b, 0) .
\end{aligned}
$$

\section{ACKNOWLEDGMENT}

The author is grateful to J. Stockman for providing waveguide simulator data and M. Zawadzki for HFSS computations. Useful discussions with Dr. R. Hodges and Dr. R. Pogorzelski are gratefully acknowledged.

\section{REFERENCES}

[1] D. M. Pozar, S. D. Targonski, and H. D. Syrigos, "Design of millimeter wave microstrip reflectarrays," IEEE Trans. Antennas Propag., vol. 45, no. 2, pp. 287-296, Feb. 1997.

[2] D. M. Pozar, "Reflectarray antenna and solar array integration for spacecraft applications," Jet Propulsion Laboratory, Pasadena, CA, Final Rep., Mar. 1997.

[3] T. Itoh, Ed., Numerical Techniques for Microwave and Millimeter-Wave Passive Structures. New York: Wiley, 1989.

[4] B. A. Munk, Frequency Selective Surfaces, Theory and Design. New York: Wiley, 2000.

[5] D. M. Pozar, "Analysis of an infinite phased array of aperture coupled microstrip patches," IEEE Trans. Antennas Propag., vol. 37, no. 4, pp. 418-425, Feb. 1989. 